Daniel Hernández-Rosete'

Olivia Maya García"

Enrique Bernal'

Xóchitl Castañeda"'I

George Lemp"II

Centro de Investigación y de Estudios Avanzados. Instituto Politécnico Nacional. Departamento de Investigaciones Educativas. Ciudad de México, México

" Universidad Nacional Autónoma de México, Ciudad de México. México

III Universitywide AIDS Research Program. University of California. California, USA

Correspondência | Correspondence: Centro de Investigación y de Estudios Avanzados

Departamento de Investigaciones Educativas Calzada Tenorios No. 235, Col. Granjas Coapa 14330 México, Distrito Federal

E-mail: drosete@ cinvestav.mx

\section{Migración y ruralización del SIDA: relatos de vulnerabilidad en comunidades indígenas de México}

\author{
Migration and ruralization of \\ AIDS: reports on vulnerability of \\ indigenous communities in Mexico
}

RESUMEN

OBJETIVO: Analizar la vulnerabilidad para ITS/VIH entre mujeres Purépechas y Zapotecas unidas en concubinato con varones que practican sexo sin protección.

MÉTODOS: Estudio etnográfico realizado en febrero del 2004 y en diciembre del 2005 en localidades indígenas de Michoacán y Oaxaca, Mexico. Estos son entidades con niveles altos de expulsión migratoria, rezago comunitario y casos registrados de VIH/SIDA. Se entrevistaron en profundidad a 91 personas: migrantes temporales (24), mujeres indígenas (33), autoridades locales (20) y personal de salud (14).

RESULTADOS: La ruralización del ITS/VIH puede relacionarse con las prácticas de iniciación sexual femeninas pero sobre todo con el miedo del migrante a que su concubina tenga relaciones extra-conyugales en su ausencia. El embarazo y la crianza son recursos masculinos de control de las esposas.

CONCLUSIONES: La migración de retorno implica formas de vulnerabilidad para las mujeres indígenas en las localidades estudiadas, cuya sexualidad tiene un remarcado carácter reproductivo. Es necesario implementar políticas de prevención para ITS/VIH dirigidas a fortalecer derechos sexuales y reproductivos de las mujeres y que tomen en cuenta aspectos de identidad sexual masculina.

DESCRIPTORES: Síndrome de inmunodeficiencia adquirida, epidemiología. Migrantes. Población indígena. Población rural. Vulnerabilidad en salud. Antropología cultural. México. 


\begin{abstract}
OBJECTIVE: To evaluate the vulnerability for STI/HIV among Mexican indigenous women in common law marriage with men who practice sex without condom.
\end{abstract}

METHODS: Ethnography study undertaken in indigenous villages of Michoacán and Oaxaca, Mexico, in February 2004 and December 2005. These rural communities are characterized by high migration rates, extreme poverty and HIV/AIDS cases. An in-depth interview was applied to transient migrants (24), indigenous women (33), local authorities (20) and health providers (14).

RESULTS: Rural propagation of STI/HIV is associated to sexual female initiation and mostly to migrants' fear their concubines will have extramarital sex during their absence. Impregnating their wives and the resulting childcare is one of the men's resources for controlling their concubines.

CONCLUSIONS: Return migration implies vulnerability for indigenous women in the rural communities studied whose sexuality has a strong reproductive profile. It is necessary to develop prevention campaigns against STI/HIV taking into account male sexual identities to improve women rights to sexual and reproductive health.

KEY WORDS: Acquired immunodeficiency syndrome, epidemiology. Transients and migrants. Indigenous population. Rural population. Health vulnerability. Anthropology, cultural. Mexico.

\section{INTRODUCCIÓN}

La migración temporal indígena mexicana hacia Estados Unidos se originó en comunidades agrícolas con problemas de rezago estructural. ${ }^{6}$ Se trata de grupos de población que históricamente han sido excluidos. Esta migración ocurrió en un contexto epidémico donde las infecciones de transmisión sexual (ITS), en particular el VIH, no sólo constituyen un problema de salud pública para ambos países, sino que también agudizan la desigualdad social al empobrecer y estigmatizar a esta población cuando enferma tanto en las localidades de origen como en las de destino.

Estudios recientes indican que la ruralización del VIH en México tiene relación con las dinámicas de circulación migratoria hacia Estados Unidos. ${ }^{10,12,14}$ Es un proceso infeccioso con dos fases. La primera ocurre durante la estancia en Estados Unidos, especialmente entre varones que viajan sin sus parejas y que tienen contactos sexuales sin condón con trabajadoras sexuales ${ }^{11,14} \mathrm{o}$ con otros hombres. ${ }^{12}$ Además se sabe que comparten jeringas contaminadas para el suministro de vitaminas, antibióticos y drogas inyectables. ${ }^{16}$
La segunda fase se presenta cuando los migrantes infectados vuelven a su localidad de origen en México ${ }^{3,11,17}$ $\mathrm{y}$, sujetos a las creencias arraigadas sobre sexualidad y control reproductivo, exigen que los contactos sexuales con las esposas o parejas que esperan en México, sean sin protección. ${ }^{8}$

Las comunidades indígenas seleccionadas tienen índices muy altos de marginación ${ }^{\mathrm{a}} \mathrm{y}$ de migración hacia Estados Unidos. ${ }^{b}$ Destaca el hecho de que presentan casos registrados ${ }^{\mathrm{c}, \mathrm{d}}$ de cuadros clínicos atribuibles al SIDA.

Las nociones de conyugalidad y nupcialidad conllevan roles sexuales definidos por medio de formas de poder asimétricas que son sostenidas por ideologías de género ${ }^{13}$ y pueden favorecer relaciones sexuales sin condón.

Son más vulnerables al VIH/SIDA las personas con precariedad de recursos jurídicos, sociales y monetarios. ${ }^{9}$ De aquí que la ruralización del VIH/SIDA en

a Ávila JL, Fuentes C, Tuirán R. Índice de marginación, 2000. Severidad de la pobreza y marginación. México: Consejo Nacional de la Población; 2002.

b Ávila JC, Tuirán R. Índices de Intensidad Migratoria México-Estados Unidos, 2000. México: Consejo Nacional de la Población; 2002. c Secretaría de Salud de Michoacán. Estadísticas de casos de VIH/SIDA acumulados de 1985 a junio de 2004 en la meseta purépecha; Morelia; 2005.

d Servicios de Salud de Oaxaca. Casos de VIH/SIDA por jurisdicción sanitaria 1986-2004. Oaxaca (México): Dirección de prevención y control de enfermedades. Oaxaca; 2004. 
México puede tener consecuencias desastrosas entre los indígenas y en general en poblaciones campesinas de México, cuya dignidad y derechos humanos suelen ser menos respetados. ${ }^{9}$

La vulnerabilidad para VIH/SIDA/ITS no depende de las preferencias sexuales, sino de escenarios donde el ejercicio violento del poder impide el uso adecuado del condón. ${ }^{5}$ Por eso, a manera de hipótesis, la vulnerabilidad para VIH/SIDA/ITS en mujeres indígenas unidas en concubinato con varones migrantes es atribuible a los procesos de vida familiar, conyugal y de identidad masculina que, regidos por el género, dificultan el uso del condón durante los ciclos de la migración de retorno.

El objetivo del presente artículo fue analizar la vulnerabilidad ante el VIH/SIDA entre mujeres indígenas unidas conyugalmente bajo el régimen de concubinato a través del robo con migrantes temporales. La finalidad es identificar necesidades de prevención, tomando en cuenta las creencias que motivan a los migrantes a no usar condón cuando regresan a México.

\section{MÉTODOS}

El trabajo de campo se realizó en dos fases. La primera en Michoacán, en febrero del 2004 en las localidades de Chilchota (Santo Tomás y Zopoco), Tangancícuaro y Ecuandureo; la segunda en Oaxaca, durante diciembre del 2004. Se entrevistaron a residentes de La Ciénega, San Pablo Huixtepec, Teotitlán del Valle y a personal de salud y representantes de agencias no gubernamentales en la ciudad de Oaxaca.

Desde el constructivismo social en su perspectiva fenomenológica ${ }^{15}$ son explorados los universos de sentido y significado que rigen el contexto cultural de las personas que participaron en el estudio. Se recurrió a la oralidad y la observación etnográfica para documentar aspectos de las prácticas sociales sobre sexualidad. Entrevistas semiestructuradas, anónimas y confidenciales fueron grabadas con el consentimiento informado de los participantes.

En total fueron 91 entrevistas entre localidades purépechas (Chilchota, Ecuandureo, Tangancícuaro, Tanaquillo y Zopoco) de Michoacán $(\mathrm{N}=41)$ y entre comunidades zapotecas de los Valles Centrales (San Pablo Huixtepec, Teotitlán del Valle y La Ciénega), en Oaxaca $(\mathrm{N}=50)$. El universo (Tabla) incluyó mujeres indígenas $(\mathrm{N}=24)$ residentes en localidades rurales de México, unidas en concubinato a través del rapto con indígenas que trabajan temporalmente en condados de California. Indígenas migrantes $(\mathrm{N}=29)$ que habían regresado a México y mujeres que habían experimentado el robo de sus hijas $(\mathrm{N}=4)$ también fueron entrevistadas. Esto permitió constituir un núcleo de informantes bilingües en español y en el idioma indígena materno. Aunque el lenguaje fue importante para considerar a los informantes como indígenas, la identidad indígena no se reduce al lenguaje pues también implica la filiación territorial, las formas de sacralidad atribuidas a los ciclos agrícolas, las fiestas patronales y el sentimiento de pertenencia a una comunidad de tipo campesino-rural. ${ }^{2}$

También se entrevistaron a personas de origen mestizo como profesores de escuelas y trabajadoras sociales $(\mathrm{N}=14)$, personal de salud, autoridades civiles y representantes de organismos no gubernamentales residentes en las localidades $(\mathrm{N}=20)$.

\section{ANALISIS DE LOS RESULTADOS}

La nupcialidad del México rural conlleva creencias y tabúes sobre sexualidad y reproducción derivadas de la

Tabla. Perfil etnográfico de entrevistados según localidades. México, 2004. N=91

\begin{tabular}{|c|c|c|}
\hline \multirow{2}{*}{ Perfil etnográfico de los informantes } & \multicolumn{2}{|c|}{$\mathrm{N}$} \\
\hline & Michoacán & Oaxaca \\
\hline \multicolumn{3}{|l|}{ Grupo I } \\
\hline $\begin{array}{l}\text { Varones Purépechas y Zapotecos, entre } 18 \text { y } 45 \text { años de edad, con experiencia de } \\
\text { residencia y/o trabajo temporal en Estados Unidos. }\end{array}$ & 10 & 14 \\
\hline \multicolumn{3}{|l|}{ Grupo II } \\
\hline $\begin{array}{l}\text { Esposas y concubinas de migrantes temporales, entre } 18 \text { y } 45 \text { años de edad, con } \\
\text { filiación Purépecha y Zapoteca. }\end{array}$ & 15 & 14 \\
\hline \multicolumn{3}{|l|}{ Grupo III } \\
\hline $\begin{array}{l}\text { Autoridades y miembros de organismos no gubernamentales con presencia en las } \\
\text { localidades. }\end{array}$ & 4 & 16 \\
\hline \multicolumn{3}{|l|}{ Grupo IV } \\
\hline $\begin{array}{l}\text { Personas que por su conocimiento y presencia en la vida pública de la comunidad, } \\
\text { puedan referir información sobre migración, cultura, VIH/SIDA y sistemas de salud } \\
\text { locales. }\end{array}$ & 11 & 3 \\
\hline \multicolumn{3}{|l|}{ Grupo V } \\
\hline Madres de mujeres unidas conyugalmente a través del rapto. & 1 & 3 \\
\hline
\end{tabular}


religiosidad católica que pueden inhibir el uso de condón. Sin embargo existen otros aspectos que refuerzan la resistencia al uso de condón. Las mujeres robadas que viven en concubinato, por ejemplo, se vuelven más vulnerables porque adquieren estatus y modos de vida donde el control familiar ejercido sobre su sexualidad dificulta el uso de condón. Esto se debe a la residencia patrilocal que resulta del arraigo de los nuevos cónyuges en el domicilio de la familia del novio.

En México el robo de la mujer con violencia ha desaparecido; persiste como un concubinato que inicia con la fuga concertada entre la mujer y su raptor. El concubinato por robo es una opción para evadir el control paterno del matrimonio, además es una estrategia de sobrevivencia ante la escasez de recursos monetarios. ${ }^{7}$ Sin embargo, como práctica de nupcialidad existe en un contexto con remarcadas desigualdades de género ya que los varones pueden iniciar su vida sexual a través del comercio sexual ${ }^{13} \mathrm{o}$ con animales de granja. En cambio, las mujeres que se unen a través del robo deben ser menores de 15 años, sin experiencia sexual previa y su virginidad es legitimada a través del ritual de sangrado femenino la noche del rapto. ${ }^{13}$ Son mujeres que inician su vida sexual con fines reproductivos, ${ }^{1}$ lo que les impide el uso de condón.

La noción de limpieza atribuida a la virginidad femenina, tiene una función simbólica tan fuerte que la mujer puede ser robada sólo cuando es virgen:

- Si te la robas y descubres que no es virgen ¿qué haces?

- Eso tendría que saberlo yo antes de traérmela.

- ¿Y si no es virgen qué pasa?

- Pues yo creo que no me la llevo a vivir conmigo.

(Varón migrante, zapoteco, soltero, 22 años, Teotitlán del Valle, Oaxaca)

La virginidad femenina no sólo es apreciada por los hombres, en las localidades investigadas es un atributo socialmente necesario para que una mujer pueda ser identificada por otras mujeres como casadera:

- Debe ir virgen una muchacha cuando se casa.... aquí sí está muy bien visto eso.

- ¿Y cuando no es así?

- Pues simplemente ya no se casa la muchacha, si ven que anda ahi, que tiene su novio y anda de loquita pues simplemente ya no se casa.

(Mujer, zapoteca, 43 años, San Pablo Huixtepec, Oaxaca)

La migración se ha convertido en un atributo de masculinidad, por eso es frecuente que cuando el varón establece un noviazgo con fines matrimoniales ya tiene experiencia migratoria. De hecho, la migración parece haber adquirido el sentido de un ritual de iniciación masculina:

"Los niños de por aquí empiezan a muy temprana edad. Se van como de 14 ó 15 años y, aunque pueden seguir estudiando, una vez que lo experimentan, la mayoría vuelve a ser migrante, reproducen el mismo esquema del padre...

(Varón migrante, purépecha, 32 años, Ecuandureo, Michoacán)

En las localidades que fueron analizadas, robarse una mujer virgen, embarazarla y haber trabajado en Estados Unidos constituyen parte del capital simbólico que interviene en la producción local de identidades masculinas. Son prácticas que transforman a los niños en "hombres verdaderos", pues así pueden ser reconocidos como tales por otros varones. El robo de una mujer virgen y la migración son recursos de legitimación masculina porque otorgan prestigio, significan medios de distinción social que dan la pauta para marcar diferencias de estatus entre hombres de la misma localidad.

\section{Del ritual de perdón a la violencia familiar}

El ritual de perdón inicia la mañana posterior a la noche del robo. Después de consumado el contacto sexual y de corroborada la virginidad de la mujer robada, la familia del raptor busca al padre de la nueva concubina para ofrecer cerveza y fruta en señal del perdón solicitado. Si el padre de la mujer raptada acepta la ofrenda se fija fecha del casamiento civil y se asume la residencia paterna del raptor como el nuevo domicilio de la mujer robada.

Cuando el ritual del perdón concluye el varón emprende la migración a Estados Unidos. Una de las consecuencias de este proceso es la estigmatización de las mujeres robadas que establecen residencia en la casa del suegro desde la noche de la fuga. Esto propicia la violencia familiar contra la raptada, quien establece una relación de subordinación que generalmente deriva en violencia psicológica y física ejercida por la suegra:

- ¿le pueden llegar a pegar a la esposa?

- Si los suegros se quedan con ella cuando el esposo se va a Estados Unidos sí.

- ¿y qué pasa con la familia de la muchacha, cómo reacciona?

- prácticamente se hace a un lado

- ¿por qué no pueden defender a la hija?

- es que si se regresa a su casa, su papá ya no la acepta, 
y es que acá piensan mucho en lo que dice la gente.

(Varón migrante, purépecha, 22 años, Santo Tomás, Michoacán)

La violencia contra las esposas de migrantes unidas en concubinato conlleva mecanismos de control que tienden a invisibilizar socialmente su magnitud:

“...las mujeres se quedan y esperan a sus maridos 10, 15 años; mientras los papás de él las vigilan de que ni salga a fiestas. Entonces la tienen totalmente vigilada y la joven se queda aqui prácticamente prisionera y pasan los días y el esposo no vuelve, y ahi está la mujer con el hijo, entonces se queda bajo el cuidado de los suegros. A veces no la dejan ni que vaya a visitar a los papás porque dicen que le van a dar malas influencias o la van a mal aconsejar."

(Jefa del Departamento Jurídico. Instituto de Atención al Migrante. Oaxaca)

La complejidad de esta violencia es que favorece riesgos de infección de VIH/ITS, pues el control de la suegra se convierte en un clima de opresión para la concubina, quien se ve sujeta a un adoctrinamiento sobre sexualidad reproductiva. Esto deriva en un orden inapelable que favorece contactos sexuales sin condón cuando el migrante regresa a México:

“... estoy aquí con mis suegros mientras que mi marido anda en Estados Unidos y ya ve que por ahi agarran otra mujer. Ahorita tiene mucho que mi esposo no ha venido, como cinco años, ya entró para seis años. $Y$ aqui estoy con mis suegros, siempre esperando... y para cuando regresa siempre viene buscando otro embarazo."

(Mujer zapoteca, 23 años, San Pablo Huixtepec, Oaxaca)

\section{Del estigma al aislamiento como vulnerabilidad}

Las parejas unidas en concubinato a través del robo son excluidas de los derechos y el reconocimiento de quienes se casan con el reconocimiento de la Iglesia. Esto propicia un orden de marginalidad porque la pareja está impedida moralmente para ocupar cargos religiosos o practicar sacramentos, como es el caso de las mayordomías o el bautismo de sus hijos.

El robo genera en la concubina un estigma que le distingue de las mujeres que están casadas por la Iglesia. Este fenómeno afecta la red social de apoyo de la concubina, lo que tiende, con los años, a envolverla en una espiral de aislamiento, dependencia económica y violencia doméstica que le colocan en situaciones que disminuyen su capacidad para prevenir y atender una infección de VIH/ITS.

El aislamiento inicia con la ruptura del vínculo con la familia de origen, sobre todo con el padre, quien generalmente cree que su honorabilidad como patriarca y jefe del grupo familiar fue humillada y que sigue estando en peligro de volver a ser deshonrado por la conducta de la hija, incluso después del rapto. Las madres de estas mujeres, en cambio, asumen posturas más flexibles:

“... cuando se robaron a mi hija pues sí sentífeo, sobre todo porque mi hija todavía no terminaba la secundaria... nomás lo que quiero es que se casen por el civil, pero como tiene apenas 16 años pues no puede hasta que cumpla los 18 años. Por eso le dije: -mira tú también, si no eres por tu voluntad allá con tus suegros, si quieres venirte, puedes regresarte pero acá vas a seguir estudiando. Pero me dice: ni modo que ahora me voy a regresar, pues ya voy a estar aquí, ahora no me voy a estar arrepintiendo."

(Madre de mujer robada, purépecha, 52 años, Santo Tomás, Michoacán)

Las tradiciones católicas sobre nupcialidad favorecen la estigmatización comunitaria de la mujer robada, sobre todo por vivir en concubinato. Es un proceso de discriminación que conduce al aislamiento social, y que continúa con el descrédito que la localidad imputa a una concubina por haber sido robada:

- ¿Con quién vive actualmente?

- Con mis suegros, vivo con ellos desde que me salí de mi casa cuando me robó mi marido. Pero ahora él viene cada dos o tres años.

- Cuando ha tenido problemas de tipo económico, ¿quién le ayuda?

- Nadie. Ya no veo ni a mi padre ni a mi madre desde que me fui a vivir con mis suegros.

- ¿Y sus hermanos?

- Mis hermanos andan en Estados Unidos. Sólo tengo una hermana, es maestra, pero no me ayuda. Asi que yo sola tengo que ver por mi y mis hijos.

(Mujer purépecha, 23 años, Ecuandureo, Michoacán)

Otro aspecto de la vulnerabilidad está ligado a la escasez de dinero. En algunas familias, las remesas son insuficientes, incluso pueden pasar varios meses antes de que se reciba algún ingreso, lo que genera endeudamiento y precariedad económica. Pero el aspecto más delicado es que la suegra asume el control de las remesas:

“... se manda el dinero a la suegra, entonces la suegra le da a la muchacha dinero para que vaya al mercado, pero el dinero lo tiene la suegra y lo gasta ella. Entonces es cuando hay problemas entre la nuera $y$ la suegra..." 
(Varón migrante, zapoteco, 30 años, Teotitlán del Valle, Oaxaca)

El abandono conyugal también es parte de la vida para las mujeres unidas en concubinato. Las temporadas que pasan los indígenas en Estados Unidos no sólo favorecen contactos sexuales casuales, sino que dan lugar a la formación de nuevos núcleos familiares:

"La gran mayoría de los que se van no se llevan a la familia, dejan a la esposa por acá, muchos tienen relaciones extramaritales y la gran mayoría se vuelve a casar. Tienen la idea de casarse por allá y dejar aquí a la esposa."

(Médico, clínica comunitaria, Ecuandureo, Michoacán)

Los relatos hablan de la escasez de recursos sociales y monetarios para enfrentar problemas de salud en general y dan cuenta de la dependencia en que viven algunas mujeres estigmatizadas por el tipo de unión conyugal, para quienes una infección de VIH podría profundizar su discriminación. Se trata de una dinámica que inicia con la muerte social atribuible al concubinato y que se agudiza a través del control que ejerce la suegra al tener dominio sobre las remesas y la vida social de la concubina, quien tiende a perder su autonomía y redes sociales.

\section{La interacción conyugal en contextos de retorno}

Los migrantes entrevistados se perciben a sí mismos como hombres ausentes en sus lugares de origen. Esto parece estar ligado a la resistencia de algunos migrantes al uso del condón con sus cónyuges en México, ya que viven con el miedo a la infidelidad de sus esposas. Los informantes creen que el embarazo de sus mujeres es un recurso confiable para mantenerlas apegadas a ellos, por eso, cuando regresan de Estados Unidos, es frecuente que busquen contactos sexuales con fines reproductivos, necesariamente sin condón:

"Conocemos familias en las que el esposo viene cada cinco años y regresa sólo para embarazar a la mujer. Viene nada más a eso, porque estando la mujer embarazada va estar quieta, tranquila, pasan tres años o cuatro años y vuelve a embarazarla para que tengan compromiso."

(Jefa del Departamento Jurídico. Instituto de Atención al Migrante, Oaxaca)

Otro recurso de control de la sexualidad femenina es la vasectomía. A diferencia de otras investigaciones, ${ }^{4}$ se detectó que no es una elección de pareja. Es decir, el miedo a la infidelidad femenina lleva a los varones a asumir la vasectomía como un secreto ante la esposa. Así, una forma de vulnerabilidad entre estas mujeres es la resistencia al uso de condón entre migrantes vasectomizados:
“... entre los migrantes que han aceptado la vasectomía, sabemos que ellos no van a poder embarazar a sus esposas. Pero en realidad es un método de vigilancia en caso de que la mujer sea fecundada por otra persona, porque en caso de embarazo el hombre vasectomizado puede alegar infidelidad femenina. Es cuando uno piensa que hay que hacer bien la vasectomía porque si fallamos la mujer puede quedar embarazada y va a ser considerada como una infidelidad."

(Directora de clínica, San Pablo Huixtepec, Oaxaca)

\section{Migración de retorno desde la narración de las mujeres}

Las mujeres entrevistadas identifican al VIH como el causante del SIDA, saben que se transmite por vía sexual y que puede prevenirse con el uso del preservativo. Sus narraciones muestran cambios en las representaciones femeninas sobre VIH/ITS, fidelidad y confianza:

“... cuando él va a regresar le pido que usemos condón. Yo no sé si ha andado con otra mujer, pues tiene sus necesidades y puede ser que vaya a casas de cita o que conquiste por ratitos."

\section{(Mujer zapoteca, 35 años, La Ciénega, Oaxaca)}

En algunos casos es posible que los migrantes acepten tener relaciones sexuales protegidas. Sin embargo, algunas mujeres mostraron dificultad para abordar el tema y más aún para decidir al respecto. De hecho algunas de las mujeres viven con miedo y angustia el regreso de sus cónyuges:

- "El otro día que me dice una señora: mi esposo no es infiel, mi esposo es bien santito, se espera hasta que llega. Créelo tu sola - le digo - porque yo no. Ya no me chupo el dedo, todos los hombres que se van y están tanto tiempo por ahí, no están solos. Siempre tienen que tener alguien por ahi aunque sea de pasadita, no exactamente para vivir con una mujer por ahi, pero si van por ahi a darse sus necesidades... Es un hecho pues, todos los hombres, sean casados, tengan hijos, no tengan hijos, de todos modos allá tienen mujeres y eso si es un problema porque nos pueden traer enfermedades".

(Mujer zapoteca, 43 años, San Pablo Huixtepec, Oaxaca)

Desde su falta de autonomía, el temor a generar un conflicto conyugal, juega un papel central en la aceptación femenina del contacto sexual sin condón:

- ¿Cómo son los encuentros sexuales cuando su esposo regresa de allá? 
- Fijese que nunca hemos platicado de eso, simplemente pues ya llegó y yo le tengo que cumplir como mujer y hasta ahí nada más, pero asi de que me ponga a platicar con él, y le diga te vas a proteger tú, pues no, lo que me voy a sacar es una regañiza y mejor digo, no, yo sé que él no anda haciendo nada malo.

(Mujer zapoteca, 35 años, San Pablo Huixtepec, Oaxaca)

Las conversaciones entre mujeres de diferentes generaciones propician el intercambio de experiencias desde relaciones de género y epidemias distintas:

- ¿Cómo es el encuentro sexual cuando regresa su pareja?

- No es lo mismo después de estar tan lejos, como que no sientes confianza y más que nada hay temor. Platico con él pero me dice que allá no tiene relaciones.

- ¿Le ha pedido usar el condón?

- Sí, pero él dice que no y me pregunta por qué no le tengo confianza. Lo hemos hecho sin condón, pero ahora ya tengo más miedo. Platico con mis tías y ellas vivieron asi también. Pero ellas me dicen que ahorita que somos jóvenes deberíamos cuidarnos, ellas me preguntan ¿tú crees que él te va a decir la verdad si sabe que te vas a enojar? O sea ellos nunca van a decir la verdad, por más confianza que les tengas. Y luego la que la lleva es una.

(Mujer purépecha, 26 años, Ecuandureo, Michoacán)

Otro relato sugiere que el miedo no sólo tiene que ver con la conciencia de que su marido la infecte, sino de que pueda quedar embarazada siendo portadora de VIH:

"Yo pienso que muchas de las que nos casamos con migrantes deberíamos exigir la prueba de SIDA a nuestros maridos porque asi puedes prevenirte para que no te embaraces".

(Mujer purépecha, 27 años, Ecuandureo, Michoacán)

La comunicación entre mujeres sugiere que los médicos comunitarios ejercen una influencia favorable para sensibilizar sobre prevención:

"La otra vez le platiqué al médico y me dijo que uno no tiene por qué arriesgar la vida, yo le pregunté al doctor si me pudiera dar algún papel para que mi esposo se haga análisis cuando regrese, porque aunque uno le tenga mucha confianza a su esposo, no hay la seguridad de que le va a ser fiel".

(Mujer purépecha, 27 años, Ecuandureo, Michoacán)

\section{CONCLUSIONES}

En México la religiosidad católica persiste como inhibidor de derechos sexuales de mujeres y hombres, especialmente con relación al uso del condón. Sin embargo, la práctica del sexo sin protección es atribuible a razones más complejas.

Los migrantes se reconocen como hombres ausentes. Este fenómeno se relaciona con la preocupación obsesiva que muestran ante el adulterio femenino. El embarazo y la crianza son vistos como recursos de control de las esposas, quienes se ven obligadas a tener sexo sin protección cuando sus maridos regresan a México. Por otro lado, la vasectomía aparece como estrategia para tratar de detectar el adulterio femenino, ya que ésta se realiza en desconocimiento de la esposa, por tanto las relaciones sexuales se practican sin condón, a fin de que un embarazo sea tenido como evidencia de infidelidad femenina.

Los varones entrevistados encuentran en las nociones de fidelidad y confianza argumentos para buscar contactos sexuales sin condón con sus esposas al regresar a México. Las mujeres, en cambio, asumen la posibilidad de que sus cónyuges hayan tenido relaciones sexuales sin protección durante su estancia en Estados Unidos y por eso reconocen que el uso de condón es una necesidad para la salud sexual y un recurso preventivo contra las infecciones, especialmente cuando tienen hijos menores de edad.

Las políticas de salud tienen que considerar que la vulnerabilidad para VIH/ITS entre las mujeres entrevistadas tiene su origen en las creencias masculinas que inhiben el uso del condón.

La ruralización del VIH/SIDA en México requiere intervenciones de prevención y atención de VIH/ITS que sean sensibles a la pluralidad etnolingüística. Además, han de estar sustentadas en enfoques de género que no sólo reconozcan a la población femenina, sino que también tomen en cuenta las prácticas y creencias masculinas sobre reproducción. Especialmente las creencias ligadas a la conversión simbólica de niños en hombres, que parecen jugar un papel importante en la producción de vulnerabilidades para VIH/ITS.

\section{AGRADECIMIENTOS}

A Soledad González de El Colegio de México(Colmex), Raquel Abrantes del Centro Interamericano de Estudios de Seguridad Social, Guillermina Natera del Instituto Nacional de Psiquiatría Ramón de la Fuente, Juan Guillermo Figueroa del COLMEX y Eduardo Weiss del Centro de Investigaciones y Estudios Avanzados Departamento de Investigaciones Educativas), por sugestiones a lo manuscripto. 


\section{REFERENCIAS}

1. Amuchástegui-Herrera A. Virginity in Mexico: the role of competing discourses of sexuality in personal experience. Reprod Health Matters.1998;6(12):105-15.

2. Bartolomé MA. Gente de costumbre y gente de razón. Las identidades étnicas en México. Siglo XXI-INI. México: Instituto Nacional Indigenista; 2004.

3. Carrier J, Magaña R. Use of ethno sexual data on men of Mexican origins for HIV/AIDS prevention programs. In: Herdt G, Lindenbaum S, editors. The Time of AIDS: Social Analysis, Theory and Method. Londres: Sage; 1992. p.243-58.

4. Castro P. ¿Qué rezones exponen los hombres que están recurriendo a la vasectomía sin bisturí para limitar su fecundidad?. En: Lerner S. Varones, sexualidad y reproducción. México: El Colegio de México; 1998; p.341-68.

5. Delor F, Hubert M. Revisiting the concept of vulnerability. Soc Sci Med. 2000;50(11):1557-70.

6. Durand, J, Massey D. El imperio dependiente. Mano de obra agrícola en Estados Unidos. En: Durand J, Massey D, editores. Clandestinos. Migración MéxicoEstados Unidos en los albores del siglo XXI. México: Grupo Editorial Miguel Ángel Porrúa; 2003; p.147-64. (Colección América Latina y el Nuevo Orden Mundial)

7. González Montes S. Las costumbres de matrimonio en el México indígena contemporáneo. En: Figueroa Campos B, coordenador. México diverso y desigual: enfoques sociodemográficos. México: Sociedad Mexicana de Demografía / El Colegio de México; 1999. p.87-141.(Investigación Demográfica en México, 4)

8. Hirsch, J, Higgins J, Bentley M, Nathanson C. The social constructions of sexuality: marital infidelity and sexually transmitted disease-HIV risk in a Mexican migrant community. Am J Public Health. 2002;92(8):1227-37.
9. Mann, J. Health and human rights: a reader. Londres: Routledge; 1998.

10. Martínez-Donate AP, Rangel MG, Hovell MF, Santibanez J, Sipan CL, Izazola JA. HIV infection in mobile populations: the case of Mexican migrants to the United States. Rev Panam Salud Publica. 2005;17(1):26-9.

11. Organista K, Carrillo H, Ayala G. HIV prevention with Mexican migrants: review, critique, and recommendations. I Acquir Immune Defic Syndr. 2004;37(Supl 4):227-39.

12. Rangel G, Martínez-Donate A, Hovell M, Santibáñez J, Sipan C, Izazola J. Prevalence risk factors for HIV infection among mexican migrants and immigrants: probability survey in the north border of Mexico. Salud Publica Mex. 2006;48(1):3-12.

13. Rodríguez G, Keijzer B. La noche se hizo para los hombres. Sexualidad en los procesos de cortejo entre jóvenes campesinos y campesinas. México; EDAMEX Population Council; 2002.

14. Sánchez MA, Lemp GF, Magis-Rodríguez C, BravoGarcía E, Carter S, Ruiz JD. The epidemiology of HIV among Mexican migrants and recent immigrants in California and Mexico. J Acquir Immune Defic Syndr. 2004;(Supl 4):S204-14.

15. Schütz A, Luckmann T. Las estructuras del mundo de la vida. Buenos Aires; Amorrortu; 2001.

16. Strathdee $S$, Fraga WD, Case $P$, Firestone $M$, Brouwer KC, Perez SG, et al. "Vivo para consumirla y la consumo para vivir" ["I live to inject and inject to live"]: high-risk injection behaviors in Tijuana, Mexico. Journal of Urban Health. 2005;82(3 Supl 4):iv58-73.

17. Zavriew L. Bringing HIV back to the villages. WorldAIDS. 1994;(35):6. 